\title{
The role of differential rotation in the evolution of the $r$-mode instability
}

\author{
Paulo M. Sa* and Brigitte Tome甘 \\ Departamento de Física and Centro Multidisciplinar de Astrofísica - CENTRA, \\ F.C.T., Universidade do Algarve, Campus de Gambelas, 8005-139 Faro, Portugal
}

\begin{abstract}
We discuss the role of differential rotation in the evolution of the $l=2 r$-mode instability of a newly born, hot, rapidly-rotating neutron star. It is shown that the amplitude of the $r$-mode saturates in a natural way at a value that depends on the amount of differential rotation at the time the instability becomes active. It is also shown that, independently of the saturation amplitude of the mode, the star spins down to a rotation rate that is comparable to the inferred initial rotation rates of the fastest pulsars associated with supernova remnants.
\end{abstract}

\section{Introduction}

The $r$-modes are non-radial pulsations modes of rotating stars that have the Coriolis force as their restoring force and a characteristic frequency comparable to the rotation speed of the star [1]. These modes are driven unstable by gravitational radiation in all rotating stars [2]. A deeper understanding of $r$-modes and their astrophysical relevance requires taking into account nonlinear effects in the evolution of the $r$-mode instability. One such a nonlinear effect, differential rotation induced by $r$-modes, has been studied by several authors. Rezzolla, Lamb and Shapiro [3] were the first to suggest that differential rotation drifts of kinematical nature could be induced by $r$-modes, deriving an approximate analytical expression for this differential rotation. Afterwards, the existence of such drifts was confirmed numerically both in general relativistic 4] and Newtonian hydrodynamics 5]. Recently, an exact $r$-mode solution, describing differential rotation of pure kinematic nature that produces large scale drifts along stellar latitudes, was found within the nonlinear theory up to second order in the mode's amplitude 6 . In this paper we discuss the role of differential rotation in the evolution of the $l=2 r$-mode instability of a newly born, hot, rapidly-rotating neutron star.

\section{The evolution model of Owen et al.}

A few years ago, Owen et al. 7] proposed a simple model to study the evolution of the $r$-mode instability in newly born, hot, rapidly-rotating neutron stars. Within this model, it is assumed that the time evolution of the system (star and $r$-mode perturbation) is characterized by two parameters: the angular velocity of the star, $\Omega(t)$, and the amplitude of the mode, $\alpha(t)$. The total angular momentum of the star is then given by

$$
J=I \Omega+J_{c}
$$

where the momentum of inertia $I$ of the equilibrium configuration is $I=\tilde{I} M R^{2}$, with $\tilde{I}=0.261$, and the canonical angular momentum $J_{c}$ of the $r$-mode perturbation is $J_{c}=-3 / 2 \alpha^{2} \Omega \tilde{J} M R^{2}$, with $\tilde{J}=1.635 \times 10^{-2}$. Here, only the $l=2 r$-mode is being considered and it is assumed that the mass density $\rho$ and the pressure $p$ of the fluid are related by a polytropic equation of state $p=k \rho^{2}$, with $k$ such that the mass and radius of the star take the values $M=1.4 M_{\odot}$ and $R=12.53 \mathrm{~km}$, respectively.

Within this model it is also assumed that the total angular momentum of the star decreases due to the emission of gravitational radiation and that the physical energy of the $r$-mode perturbation (in the co-rotating frame) increases due to the emission of gravitational radiation and decreases due to the dissipative effect of viscosity. These assumptions lead then to a system of differential equations for the angular momentum of the star, $\Omega$, and the amplitude of the $r$-mode, $\alpha$ :

$$
\begin{aligned}
\frac{d \Omega}{d t} & =-\frac{2 \Omega}{\tau_{V}} \frac{Q \alpha^{2}}{1+Q \alpha^{2}}, \\
\frac{d \alpha}{d t} & =-\frac{\alpha}{\tau_{G R}}-\frac{\alpha}{\tau_{V}} \frac{1-Q \alpha^{2}}{1+Q \alpha^{2}},
\end{aligned}
$$

where $Q=3 \tilde{J} /(2 \tilde{I})=0.094$, the gravitational-radiation and viscous timescales, $\tau_{G R}$ and $\tau_{V}$, are given by [8]

$$
\begin{aligned}
\frac{1}{\tau_{G R}} & =\frac{1}{\tilde{\tau}_{G R}}\left(\frac{\Omega^{2}}{\pi G \bar{\rho}}\right)^{3}, \\
\frac{1}{\tau_{V}} & =\frac{1}{\tilde{\tau}_{S}}\left(\frac{10^{9} \mathrm{~K}}{T}\right)^{2}+\frac{1}{\tilde{\tau}_{B}}\left(\frac{T}{10^{9} \mathrm{~K}}\right)^{6}\left(\frac{\Omega^{2}}{\pi G \bar{\rho}}\right) .
\end{aligned}
$$

In the above expressions, the fiducial timescales are $\tilde{\tau}_{G R}=-3.26 \mathrm{~s}, \tilde{\tau}_{S}=2.52 \times 10^{8} \mathrm{~s}$ and $\tilde{\tau}_{B}=6.99 \times 10^{8} \mathrm{~s}$.

For a newly born, hot, rapidly-rotating neutron star there is an interval of relevant temperatures and angular velocities of the star for which the gravitational timescale is much smaller than the viscous timescale, $\tau_{\mathrm{GR}} \ll \tau_{\mathrm{V}}$. Therefore, for this interval of temperatures and angular velocities, we can neglect in the right-hand side of Eqs. (2) and (3) the terms proportional to $\tau_{V}^{-1}$ and obtain the solution $\Omega=\Omega_{0}$ and $\alpha=\alpha_{0} \exp \left\{-\left(t-t_{0}\right) / \tau_{G R}\right\}$. If the initial angular velocity is chosen to be $\Omega_{0}=\Omega_{K}$, where $\Omega_{K}=(2 / 3) \sqrt{\pi G \bar{\rho}}$ is the Keplerian angular velocity at which the star starts shedding mass at the equator, then $\tau_{G R}=-37.1 \mathrm{~s}$, implying that the perturbation
*Electronic address: pmsa@ualg.pt

${ }^{\dagger}$ Electronic address: btome@ualg.pt 
grows exponentially from an initial amplitude $\alpha_{0}=10^{-6}$ to values of order unity in just about $500 \mathrm{~s}$ [7]. After this first stage of evolution of the $r$-mode instability, the amplitude $\alpha$ has to be forced, by hand, to take a certain saturation value $\alpha_{\text {sat }} \leqslant Q^{-1 / 2}=3.26$; in the second stage of the evolution it is then assumed that $\alpha=\alpha_{\text {sat }}$ and $\Omega$ is determined from the equation

$$
\frac{d \Omega}{d t}=\frac{2 \Omega}{\tau_{G R}} \frac{\alpha_{s a t}^{2} Q}{1-\alpha_{s a t}^{2} Q},
$$

which yields the solution

$$
\Omega(t)=\Omega_{0}\left[\frac{0.030 \alpha_{\text {sat }}^{2}}{1-Q \alpha_{\text {sat }}^{2}}\left(\frac{\Omega_{0}}{\Omega_{K}}\right)^{6}\left(\frac{t-t_{*}}{\sec }\right)+1\right]^{-1 / 6}
$$

where $t_{*}$ is the time at which occurs the transition from the first to the second stage of evolution. During the second stage of evolution, the star loses its angular momentum, spinning down to its final angular velocity. As can be seen from the above equations, the final angular velocity of the star depends critically on the saturation value of the mode's amplitude $\alpha_{\text {sat }}$; for instance, after one year of evolution, for $\Omega_{0}=\Omega_{K}$ and $\alpha_{\text {sat }}=1$ one obtains $\Omega \simeq 0.1 \Omega_{K}$, while for $\alpha_{\text {sat }}=10^{-3}$ the angular velocity is $\Omega \simeq 0.9 \Omega_{K}$. The fact that the growth of the mode's amplitude has to be stopped by hand at a certain saturation value introduces an element of arbitrariness into the solution, permitting, for instance, that agreement between the predicted final value of the angular velocity of the star and the value inferred from astronomical observations can always be achieved by fine-tuning the value of the saturation amplitude. As will be seen in the next section, this arbitrariness disappears when differential rotation is taken into account.

\section{Evolution model with differential rotation}

Differential rotation induced by $r$-modes does contribute to the physical angular momentum of the $r$-mode perturbation [6]. Therefore, a model for the evolution of the $r$-mode instability should include the effect of differential rotation. Here, we adopt the model of Owen et al. [7], described in the previous section, with the important difference $^{1}$ that the total angular momentum of the star is given by

$$
J=I \Omega+\delta^{(2)} J
$$

where the physical angular momentum of the $r$-mode perturbation $\delta^{(2)} J$ is $[\underline{6}$

$$
\delta^{(2)} J=\frac{1}{2} \alpha^{2} \Omega(4 K+5) \tilde{J} M R^{2} .
$$

\footnotetext{
1 There is another difference, albeit less significant, between the model of Owen et al. 7] and the model we adopt here, namely, we deduce the evolution equations just from angular momentum considerations.
}

In the previous expression, $K$ is a constant fixed by the choice of initial data and gives the initial amount of differential rotation associated with the $r$-mode. The specific case $K=-2$, for which the physical angular momentum of the $r$-mode perturbation coincides with the canonical angular momentum, corresponds to the model of Owen et al. 7] described in great detail in the previous section. There is not, to our knowledge, any physical condition that forces $K$ to take such a particular value $K=-2$. Therefore, we will consider in this paper arbitrary values of $K$ in the interval $-5 / 4 \leqslant K \ll 10^{132}$.

Following the procedure described in the previous section, we arrive at a system of two first-order coupled differential equations determining the time evolution of the amplitude of the $r$-mode $\alpha(t)$ and of the angular velocity of the star $\Omega(t)$ :

$$
\begin{aligned}
& \frac{d \Omega}{d t}=\frac{8}{3}(K+2) Q \frac{\Omega \alpha^{2}}{\tau_{G R}}, \\
& \frac{d \alpha}{d t}=-\left[1+\frac{4}{3}(K+2) Q \alpha^{2}\right] \frac{\alpha}{\tau_{G R}},
\end{aligned}
$$

valid when the damping effect of viscosity can be neglected relatively to the driving effect of gravitational radiation.

This system of equations was solved analytically and discussed in great detail in Ref. [9]. In the initial stages of the evolution of the $r$-mode instability $\alpha$ increases exponentially [9],

$$
\alpha(t) \simeq \alpha_{0} \exp \left\{0.027\left(\frac{\Omega_{0}}{\Omega_{K}}\right)^{6}\left(\frac{t-t_{0}}{\mathrm{sec}}\right)\right\} ;
$$

while for later times, $\alpha$ increases very slowly as

$$
\alpha(t) \simeq 2.48\left(\frac{\Omega_{0}}{\Omega_{K}}\right)^{3 / 5}\left(\frac{t-t_{0}}{\sec }\right)^{1 / 10} \frac{1}{\sqrt{K+2}} .
$$

The amplitude of the mode saturates in a natural way and a smooth transition between the regimes (12) and (13) occurs for $t-t_{0} \simeq$ few $\times 10^{2}$ seconds (see Fig. (1). This contrasts with the model of Owen et al. 7], in which, after the short initial period of exponential growth, the amplitude $\alpha$ has to be forced by hand to take a certain saturation value $\alpha_{\text {sat }} \leqslant Q^{-1 / 2}$. As can be seen from Eq. (13), the saturation value of the amplitude of the mode depends crucially on the parameter $K$, namely, $\alpha_{\text {sat }} \propto(K+2)^{-1 / 2}$. If $K \simeq 0$, corresponding to a situation in which the initial amount of differential rotation is

\footnotetext{
2 The upper limit for $K$ results from the fact that one wishes to impose the condition that $\left|\delta^{(2)} J\left(t_{0}\right)\right| \ll I \Omega_{0}$ (the initial amplitude $\alpha_{0} \equiv \alpha\left(t_{0}\right)$ is considered throughout the paper to be $\left.10^{-6}\right)$. The lower limit for $K$ results from the fact that we do not want to saturate the amplitude of the mode by hand, a procedure needed for the case $K<-5 / 4$ in order to avoid that the total angular momentum of the star becomes negative.
} 


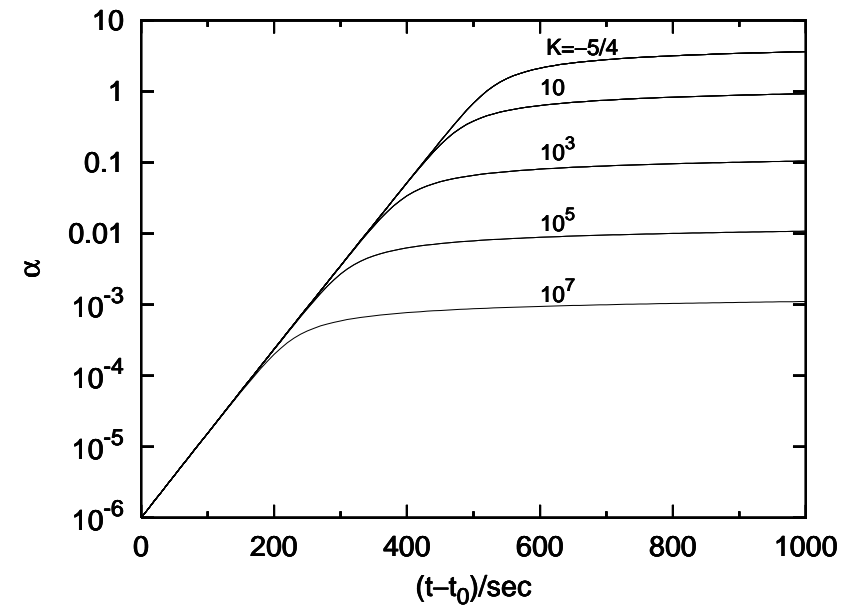

FIG. 1: Time evolution of the amplitude of the $r$-mode for different values of $K$. The initial values of the amplitude of the mode and of the angular velocity of the star are, respectively, $\alpha_{0}=10^{-6}$ and $\Omega_{0}=\Omega_{K}$.

small, then the $r$-mode saturates at values of order unity. If, on the other hand, the initial differential rotation associated to $r$-modes is significant, then the saturation amplitude $\alpha_{\text {sat }}$ can be as small as $10^{-3}-10^{-4}$.

Let us now turn our attention to the time evolution of the angular velocity of the star, $\Omega$. In the initial stages of the evolution of the $r$-mode instability $\Omega$ decreases as 9]

$\frac{\Omega(t)}{\Omega_{0}} \simeq 1-\frac{4}{3}(K+2) Q \alpha_{0}^{2} \exp \left\{0.054\left(\frac{\Omega_{0}}{\Omega_{K}}\right)^{6}\left(\frac{t-t_{0}}{\mathrm{sec}}\right)\right\} ;$

while for later times $\Omega$ decreases slowly as

$$
\frac{\Omega(t)}{\Omega_{0}} \simeq 1.30\left(\frac{\Omega_{0}}{\Omega_{K}}\right)^{-6 / 5}\left(\frac{t-t_{0}}{\sec }\right)^{-1 / 5} .
$$

The smooth transition between the regimes (14) and (15) occurs for $t-t_{0} \simeq$ few $\times 10^{2}$ seconds (see Fig. 21). Remarkably, in the later phase of the evolution, the angular velocity $\Omega$ does not depend on the value of $K$ and, consequently, does not depend on the saturation value of $\alpha$. This contrasts with the results obtained in Ref. [7], where the value of $\Omega$ depends critically on the choice of $\alpha_{\text {sat }}$.

After about one year of evolution, when the dissipative effect of viscosity becomes dominant and starts damping the mode, the angular velocity of the star becomes $\Omega_{\text {one year }} \simeq 0.042 \Omega_{K}$ (for $\Omega_{0}=\Omega_{K}$ ), in good agreement with the inferred initial angular velocity of the fastest pulsars associated with supernova remnants.

\section{Conclusions}

In this paper we have discussed the role of differential rotation in the evolution of the $l=2 r$-mode instabil-

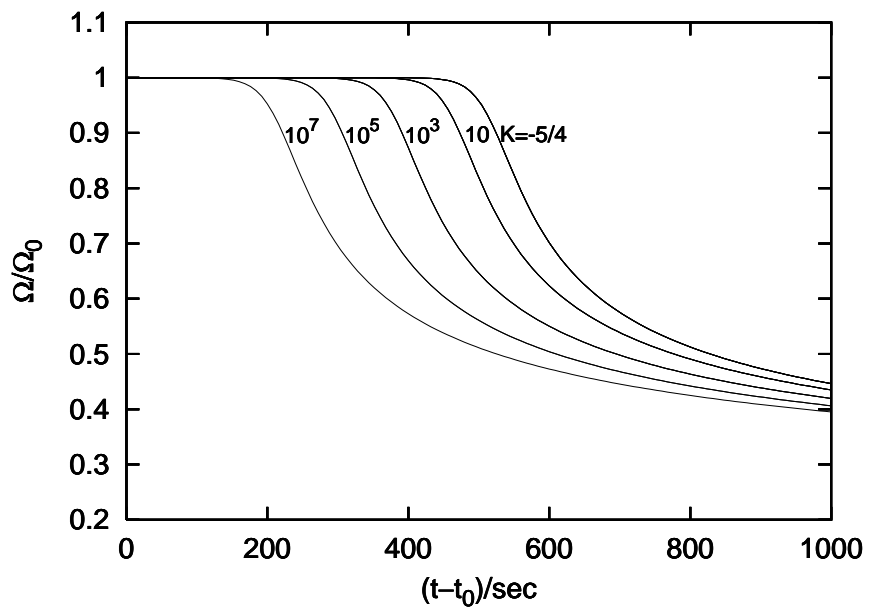

FIG. 2: Time evolution of the angular velocity of the star for different values of $K$. The initial values of the amplitude of the mode and of the angular velocity of the star are, respectively, $\alpha_{0}=10^{-6}$ and $\Omega_{0}=\Omega_{K}$.

ity of a newly born, hot, rapidly-rotating neutron star. We have shown that, a few hundred seconds after the mode instability sets in, the amplitude of the $r$-mode saturates in a natural way at values that depend on the initial amount of differential rotation associated to the $r$-mode. If the initial differential rotation of $r$-modes is small, then the $r$-mode saturates at values of order unity. On the other hand, if the initial differential rotation is significant, then the saturation amplitude can be as small as $10^{-3}-10^{-4}$. These low values for the saturation amplitude of $r$-modes are of the same order of magnitude as the ones obtained in recent investigations on wind-up of magnetic fields [3] and on nonlinear modemode interaction [10]. We have also shown that the value of the angular velocity of the star becomes, after a short period of evolution of the $r$-mode instability, very insensitive to the saturation value of the mode's amplitude. After about one year of evolution the angular velocity is $0.042 \Omega_{K}$ (for any $\alpha_{\text {sat }}$ ), in good agreement with the inferred initial angular velocity of the fastest pulsars associated with supernova remnants.

\section{Acknowledgments}

We thank Óscar Dias and Luciano Rezzolla for helpful discussions. This work was supported in part by the Fundação para a Ciência e a Tecnologia (FCT), Portugal. BT acknowledges financial support from FCT through grant PRAXIS XXI/BD/21256/99. 
[1] J. Papaloizou and J. E. Pringle, Mon. Not. R. Astron. Soc. 182, 423 (1978).

[2] N. Andersson, Astrophys. J. 502, 708 (1998).

[3] L. Rezzolla, F. K. Lamb and S. L. Shapiro, Astrophys. J. 531, L139 (2000).

[4] N. Stergioulas and J. A. Font, Phys. Rev. Lett. 86, 1148 (2001).

[5] L. Lindblom, J. E. Tohline and M. Vallisneri, Phys. Rev. Lett. 86, 1152 (2001).

[6] P. M. Sá, Phys. Rev. D. 69, 084001 (2004).
[7] B. J. Owen, L. Lindblom, C. Cutler, B. F. Schutz, A. Vecchio and N. Andersson, Phys. Rev. D. 58, 084020 (1998).

[8] L. Lindblom, B. J. Owen and S. M. Morsink, Phys. Rev. Lett. 80, 4843 (1998).

[9] P. M. Sá and B. Tomé, Phys. Rev. D 71, 044007 (2005).

[10] P. Arras, E. E. Flanagan, S. M. Morsink, A. K. Schenk, S. A. Teukolsky and I. Wasserman, Astrophys. J. 591, 1129 (2003). 